\title{
Revision of the JENDL FP Fission Yield Data
}

\author{
Jun-ichi Katakura ${ }^{1, a}$, Futoshi Minato ${ }^{2}$, and Kazuya Ohgama ${ }^{3}$ \\ ${ }^{1}$ Nagaoka University of Technology, 1603-1 Kamitomioka, Nagaoka, Niigata, Japan \\ ${ }^{2}$ Japan Atomic Energy Agency, Nuclear Science and Engineering Center, 2-4, Shirakata, Tokai-mura, \\ Ibaraki, Japan \\ ${ }^{3}$ Japan Atomic Energy Agency, Advanced Fast Reactor Cycle System R\&D Center, 4002, Narita, O-arai, \\ Ibaraki, Japan
}

\begin{abstract}
Some fission yields data of JENDL FP Fission Yields Data File 2011 (JENDL/FPY-2011) revealed inadequacies when applied to delayed neutron related subjects. The sensitivity analyses of decay heat summation calculations also showed some problems. From these results the fission yields of JENDL/FPY-2011 have been revised. The present report describes the revision of the yield data by emphasizing the sensitivity analyses.
\end{abstract}

\section{Introduction}

The JENDL/FPY-2011 file [1] was released in 2011. After the release of the file, several feedbacks from the users of the file were reported. One of them is from delayed neutron application [2] where it was shown that the yield of ${ }^{86} \mathrm{Ge}$ played significantly. The sensitivity analyses of decay heat summation calculation also showed inadequacies of some fission yield data.

The sensitivity analysis method of decay heat summation calculation has been developed [3] for the estimation of the uncertainties emerging from nuclear data used. In the course of the sensitivity analyses of fast neutron fission, the comparison of the uncertainties between fast and thermal neutron fissions was performed and it was found that the uncertainties of the calculated decay heat for thermal neutron fission were unexpectedly larger than those for fast neutron fission at short cooling times after a fission burst when the JENDL/FPY-2011 file was employed. During the search for the reason, it was found that the fission yields of the nuclides contributing to the large uncertainties of the decay heat calculations seemed to be too large. The yield data of the nuclides which showed unexpectedly large values were revised.

In the present paper, the comparison of the uncertainties between the thermal and the fast neutron fissions and the revision of the JENDL yield data are described.

\section{Comparison of decay heat uncertainties for thermal and fast neutron fission}

The uncertainties of decay heat summation calculations were derived for thermal and fast neutron fissions using JENDL FP Decay Data File 2011 (JENDL/FPD-2011) and JENDL/FPY-2011. The

ae-mail: j_katakura@vos.nagaokaut.ac.jp 
total uncertainties of the calculated decay heat values at short cooling times after a fission burst are shown in Figure 1. In this figure, the results of ${ }^{235} \mathrm{U}$ and ${ }^{239} \mathrm{Pu}$ fissions are shown. In the case of ${ }^{239} \mathrm{Pu}$

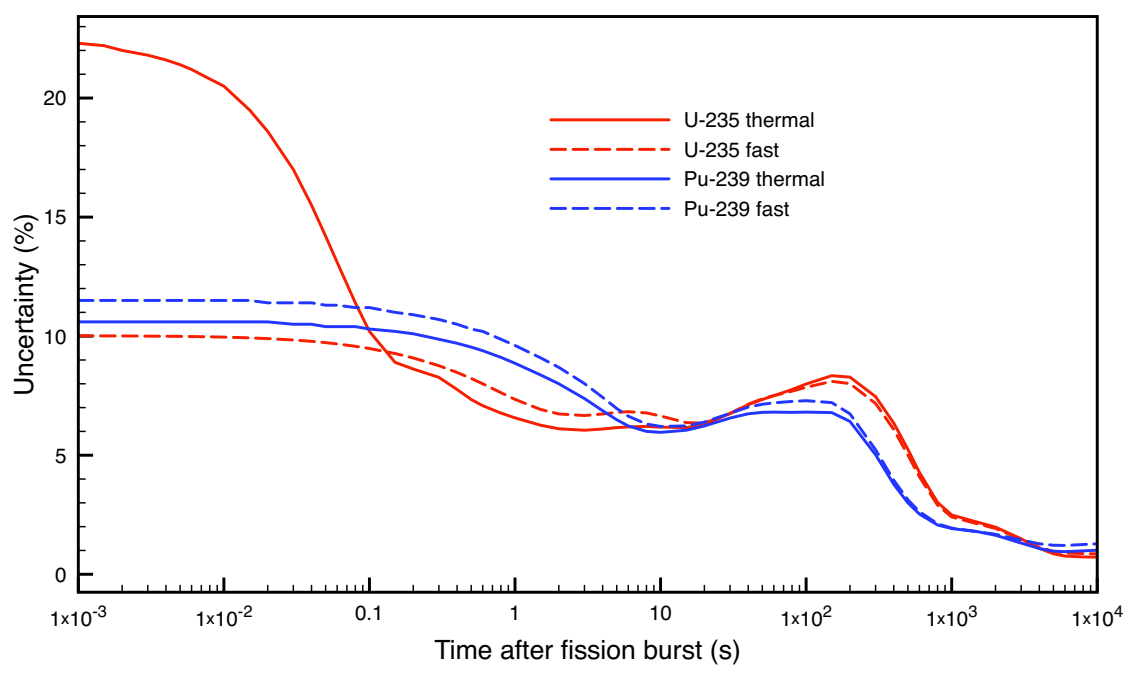

Figure 1. Uncertainties of decay heat summation calculations for thermal and fast neutron fissions using JENDL/FPD-2011 and JENDL/FPY-2011.

fission, the uncertainties of the fast neutron fission and the thermal neutron fission are not so different from each other. However, in the case of ${ }^{235} \mathrm{U}$ fission, the uncertainties of the thermal neutron fission are much larger than those of the fast neutron fission at shorter cooling times than $0.1 \mathrm{~s}$. The nuclides contributing to the uncertainties at the short cooling times are shown in Figure 2 as a time depending sensitivity. On the left-hand side, the nuclides with large sensitivity for ${ }^{235} \mathrm{U}$ thermal neutron fission are indicated. On the right-hand side, those for ${ }^{235} \mathrm{U}$ fast neutron fission are indicated. As seen in the figures, the nuclide of ${ }^{86} \mathrm{Ge}$ has large contribution to the thermal fission but no significant contribution to the fast neutron fission. The contributions of ${ }^{88} \mathrm{As}$ and ${ }^{100} \mathrm{Rb}$ are not significant but sizable contribution to the uncertainties of the ${ }^{235} \mathrm{U}$ thermal neutron fission and no contribution to
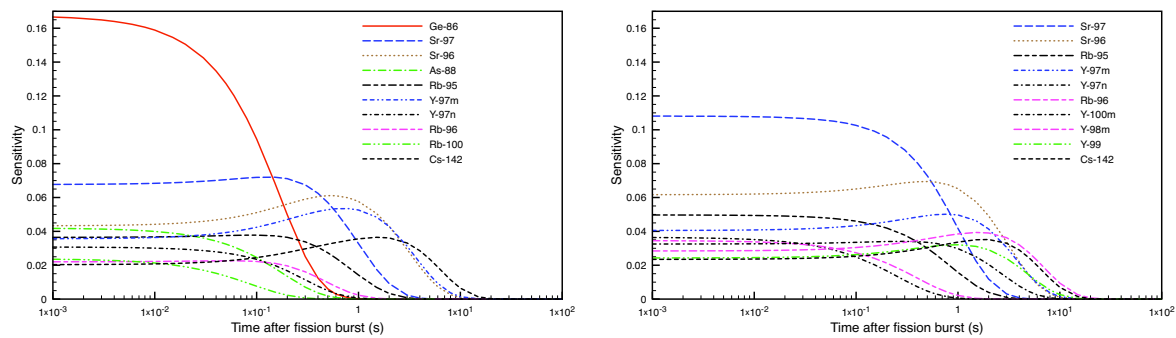

Figure 2. Important sensitivity coefficients at short cooling times after fission. 
those of the fast neutron fission. The independent fission yields of those nuclides for the thermal neutron fission of ${ }^{235} \mathrm{U}$ in JENDL/FPY-2011 are listed in Table 1. As the fission yields depend on

Table 1. Comparison of independent fission yields of JENDL/FPY-2011.

\begin{tabular}{|c|c|c|c|}
\hline Nuclide & Thermal & Fast & Ratio (Fast/Termal) \\
\hline${ }^{86} \mathrm{Ge}$ & $6.28 \times 10^{-3}$ & $5.41 \times 10^{-6}$ & $8.61 \times 10^{-4}$ \\
${ }^{88} \mathrm{As}$ & $1.24 \times 10^{-3}$ & $2.01 \times 10^{-5}$ & $1.62 \times 10^{-2}$ \\
${ }^{100} \mathrm{Rb}$ & $3.47 \times 10^{-4}$ & $1.55 \times 10^{-7}$ & $4.45 \times 10^{-4}$ \\
\hline
\end{tabular}

incident neutron energy, it is true that the yields are different from each other for thermal and fast fission. However, the differences shown in Table 1 seem to be too large. The charge distribution of the same masses as those nuclides, that is, $\mathrm{A}=86,88$ and 100 are shown in Figure 3. From Figure 3
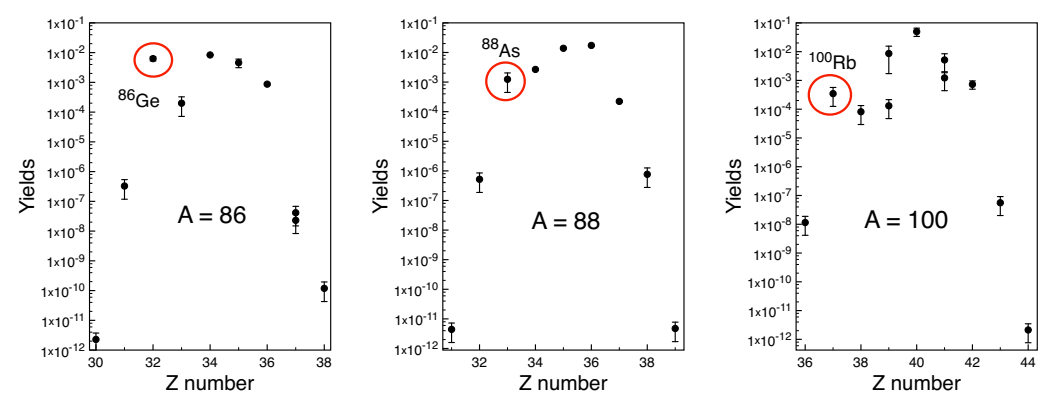

Figure 3. Charge distribution of fission yields for $\mathrm{A}=86,88$ and 100 .

the yields corresponding to ${ }^{86} \mathrm{Ge}(\mathrm{Z}=32$ for $\mathrm{A}=86),{ }^{88} \mathrm{As}(\mathrm{Z}=33$ for $\mathrm{A}=88)$ and ${ }^{100} \mathrm{Rb}(\mathrm{Z}=37$ for $A=100$ ) look like abnormally large comparing with other yields.

\section{Fission yields of JENDL/FPY}

The fission yield data of JENEL/FPY-2011 are based on the evaluation of ENDF/B-VI [4]. Although some modifications were carried out for the compilation of the JENDL file because of the differences of decay chains and isomers adopted in the JENDL and ENDF files, basically the independent yields of the JENDL file were taken from those of ENDF/B-VI. As for the ${ }^{86} \mathrm{Ge}$ case, the problem of the ENDF yield data was claimed previously [5]. And recent analysis of delayed neutron also pointed out that the yield data of ${ }^{86} \mathrm{Ge}$ of JENDL/FPY-2011 severely affect the reactivity analysis of a reactor [2]. The independent fission yields of JENDL and ENDF are listed in Table 2. As pointed out above, the yield value of the JENDL file is similar to that of ENDF.

As the ENDF/B-VI yield data are based on the reported ones in the report of LA-UR-94-3106 [4] which contains detailed data sources used for the evaluation work. In the appendix A of the report, the measured cumulative yield of ${ }^{86} \mathrm{Ge}$ by ${ }^{235} \mathrm{U}$ thermal neutron fission is listed to be $0.87 \pm 0.12 \%$ as listed in Figure 4. The red line shows the yield of ${ }^{86} \mathrm{Ge}$ with the reference of 90rud1. The reference designated as 90rud1 is Radiochimica Acta 49, 155-191 (1990) [6]. However, the value of $0.87 \pm 0.12$ for ${ }^{86} \mathrm{Ge}$ is not found in the original paper. There is no yield data of ${ }^{86} \mathrm{Ge}$ in the paper. The isotope 
Table 2. Independent yields of ${ }^{86} \mathrm{Ge}$ in ENDF/B-VI and JENDL/FPY.

\begin{tabular}{|c|c|}
\hline ENDF/B-VI & JENDL/FPY-2011 \\
\hline $6.28666 \times 10^{-3}$ & $6.27711 \times 10^{-3}$ \\
\hline
\end{tabular}

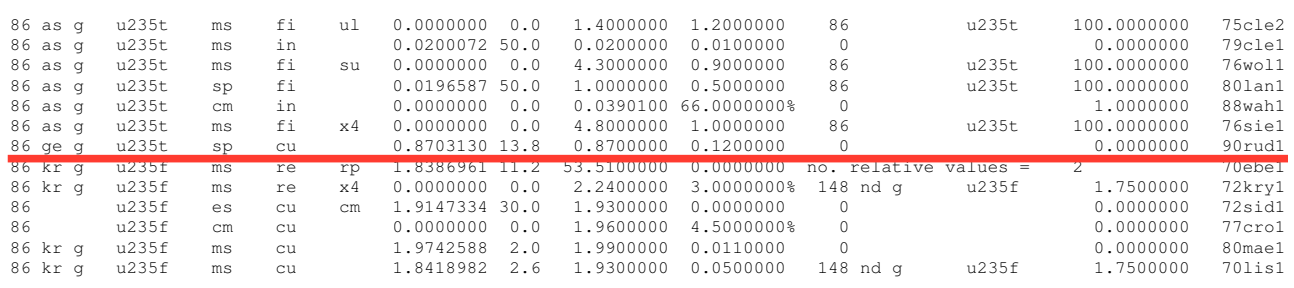

Figure 4. Measured data extracted from Appendix A of LA-UR-94-3106.

whose measured yield is $0.87 \pm 0.12 \%$ is ${ }^{86} \mathrm{Se}$, not ${ }^{86} \mathrm{Ge}$. So the value of the appendix A of LA-UR94-3106 seems to be incorrectly cited. As the cumulative yield of ${ }^{86} \mathrm{Se}$ seems to be mistaken to be that of ${ }^{86} \mathrm{Ge}$, the independent yield of ${ }^{86} \mathrm{Ge}$ would be also adjusted to give the mistaken cumulative yield. As for other nuclide yields, similar mistakes were considered to have happened. Then the independent yields of the nuclides mentioned above were discarded and newly calculated to give new yield data of JENDL.

\section{Calculation of independent yield}

The independent fission yields were calculated following the equations in the report of JENDL/FPD2011 [3]. The independent yield $y_{i}(A, Z)$ of a nuclide with charge $\mathrm{Z}$ and mass $\mathrm{A}$ is expressed by:

$$
y_{i}(A, Z)=Y(A) \times f(A, Z) \times F_{x}(A, Z) \times r_{i}(A, Z),
$$

where $Y(A)$ is the mass yield, $f(A, Z)$ the fractional independent yield, $F_{x}(A, Z)$ the pairing and oddeven correction factor and $r_{i}(A, Z)$ the isomeric yield ratio.

The fractional independent yield $f(A, Z)$ was calculated by:

$$
f(A, Z)=\frac{N(A)}{\sigma_{A} \sqrt{2 \pi}} \int_{Z-0.5}^{Z+0.5} \exp \left[-\frac{\left(Z-Z_{p}(A)\right)^{2}}{2 \sigma_{A}^{2}}\right] d Z,
$$

where $Z_{p}(A)$ is the most probable charge of mass $A, \sigma_{A}$ the Gaussian width of mass $\mathrm{A}$ and $N(A)$ the normalization factor. The normalization factor $N(A)$ is given as the summation of all elements of each mass becomes 1.0.

$$
\sum_{Z=0}^{\infty} f(A, Z) \cdot F_{x}(A, Z)=1.0 .
$$

The value of $Z_{p}(A)$ and $\sigma_{A}$ were taken from LA-UR-94-3106.

The new charge distributions of $A=86,88$ and 100 are shown in Figure 5. Comparing with Figure 3, the abnormal behaviors of ${ }^{86} \mathrm{Ge},{ }^{88} \mathrm{As}$ and ${ }^{100} \mathrm{Rb}$ seem to be disappeared. The new values were compared with those of the JEFF evaluated values [7], too. The new values and those of JEFF evaluation are listed in Table 3. It is found out that the new JENDL values are same order as those of JEFF. 

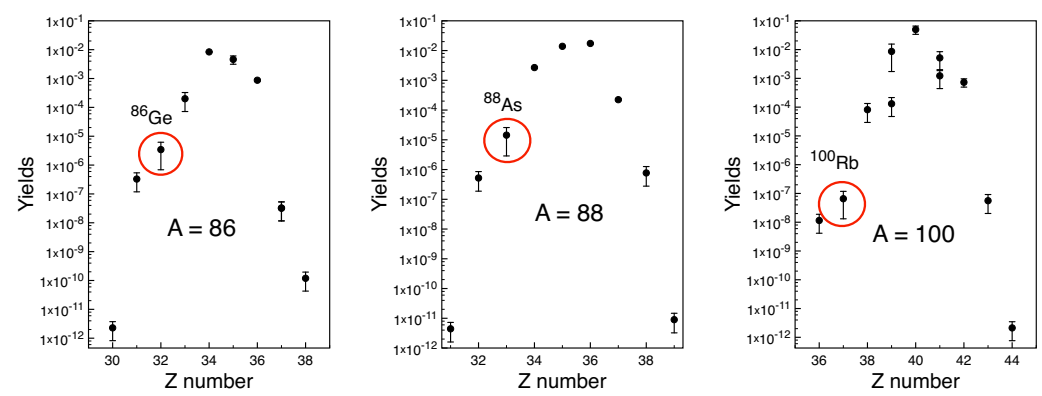

Figure 5. Charge distribution of new fission yields for $\mathrm{A}=86,88$ and 100 .

Table 3. New independent yields of JENDL and those of JEFF.

\begin{tabular}{|c|c|c|c|}
\hline & ${ }^{86} \mathrm{Ge}$ & ${ }^{88} \mathrm{As}$ & ${ }^{100} \mathrm{Rb}$ \\
\hline New JENDL & $3.4405 \times 10^{-6}$ & $1.4342 \times 10^{-5}$ & $6.5699 \times 10^{-8}$ \\
JEFF 3.1.1 & $2.8497 \times 10^{-6}$ & $1.0704 \times 10^{-5}$ & $3.9483 \times 10^{-8}$ \\
\hline
\end{tabular}

\section{Summary}

Sensitivity analysis of decay heat summation calculation showed some discrepancies between the uncertainties of thermal and fast neutron fissions for ${ }^{235} \mathrm{U}$ when the JENDL/FPY-2011 and the JENDL/FPD-2011 files. It was found that the several fission yields of the JENDL/FPY-2011 file had unexpectedly large and abnormal discontinuity of charge distribution. Those fission yields were updated. The updated yield data seem to remove the abnormal discontinuation of the charge distribution and to show rather smooth distribution. The sensitivity analysis is useful tool to analyze the uncertainty of decay heat calculation and to improve the data quality. The deficiencies of the fission yields were also suggested by the feedbacks from the users of the JENDL file. The user feedback is also important for improving the quality of the evaluated data.

\section{References}

[1] J. Katakura, "JENDL FP Decay Data File 2011 and Fission Yields Data File 2011", JAEAData/Code 2011-025, Japan Atomic Energy Agency (2011).

[2] G. Chiba, M. Tsuji, and T. Narabayashi, Nucl. Data Sheets, 118, 401-404 (2014).

[3] J. Katakura, J. Nucl. Sci. Technol., 50, 799-807 (2013).

[4] T.R. England and B.F. Rider, "Evaluation and Compilation of Fission Product Yields", LA-UR94-3106, ENDF-349, Los Alamos National Laboratory (1994).

[5] K. Oyamatsu, J. Nucl. Sci. Technol. Supp. 2, 1109 (2002).

[6] G. Rudstam, P. Aagaard, B. Ekstrom, E. Lund, H. Gokturk, H.U. Zwicky, Radiochimica Acta 49, 155-191 (1990).

[7] M.A. Kellet, O. Bersillon and R.W. Mills, "The JEFF-3.1/-3.1.1 radioactive decay data and fission yields sub-libraries”, JEFF Report 20, OECD/NEA Data Bank (2009). 
